\title{
Offline Signature Verification using FMCN with GA based Optimization of Features
}

\author{
Yogendra Kumar Jain \\ HOD, Computer Sc. \& Engineering Department \\ Samrat Ashok Technological Institute \\ Vidisha (M.P.) India
}

\author{
Rupal S. Patil \\ Computer Sc. \& Engineering Department \\ Samrat Ashok Technological Institute \\ Vidisha (M.P.) India
}

\begin{abstract}
In recent years, along with extraordinary diffusion of internet and growing need of personal identification in many applications, signature verification is considered with interest. This paper proposed an offline signature verification method based on Genetic Algorithm and Fuzzy Min Max Neural Network Classifier with Compensatory Neuron. The proposed method is basically consists of two steps. At first step optimizing the features using genetic algorithm, and at second step signature recognition is done using Fuzzy Min Max Neural Network Classifier with Compensatory Neurons. The sample of signatures is used to represent a particular person. The sample signature is first preprocessed, and then features of the processed signature are extracted by using Krawtchouk moment. After feature extraction, these features are optimized by using genetic algorithm and finally optimized features are given to the classification phase for recognition. With this proposed method, we get the $98 \%$ accuracy in recognition and less time is required for classification with optimized features as compared to time required for classification without optimizing feature.
\end{abstract}

\section{Keywords}

Signature Verification, Krawtchouk Moment Invariant Genetic Algorithm, Fuzzy Min Max Neural Network with Compensatory Neuron

\section{INTRODUCTION}

The security requirements of the today's society have placed biometrics at the center of a large debate, as it is becoming a key aspect in a multitude of applications. The term biometrics refers to individual recognition based on a person's distinguishing characteristics. Signature recognition system is classified as online and offline system. In online, signatures are acquired during the writing process with a special instrument, such as pen tablet. Dynamic information is always available in case of online signature recognition, such as velocity, acceleration and pen pressure which is more difficult to estimate than the Static shape of signature [1]. For the offline signature recognition systems [2] [3] [4], the previously written signature are captured by scanning or by other biometric system as a static image and then the recognition is carried out.

Handwritten signatures occupy a very special place in this wide set of biometric traits [5], [6], [7], [8], [9]. This is mainly due to the fact that handwritten signatures have long been established as the most widespread means of personal verification. A handwritten signature is the result of a complex process depending on the psychophysical state of the signer and the conditions under which the signature apposition process occurs.

The main phases for signature verification system adopted in the literature are Data Acquisition, Preprocessing of data (signatures), Feature Extraction and Classification. In this paper, we have taken one more phase as Feature Optimization. The main advantage of using Feature Optimization phase is less learning time required for Classification.

In Data Acquisition, the database of signature is generated. In preprocessing gray scaling, binarization and signature outline operations are performed. After preprocessing, the features of the signature are extracted using Krawtchouk Moment Invariant and stored as the finite vectors. After Feature Extraction, the features are optimized by using Genetic Algorithm so that the features provided for classification phase is less and hence the learning time required for classification will be less. The final phase is the classification. In this phase, training and testing of the signature with the available database is done.

\section{BACKGROUND}

\subsection{Preprocessing}

Signature samples are captured by the scanner and conditioned to get required signature pattern for feature extraction using preprocessing technique. The preprocessing includes gray scaling, binarization and signature outline calculation. The preprocessing steps have been presented in Figure 2.
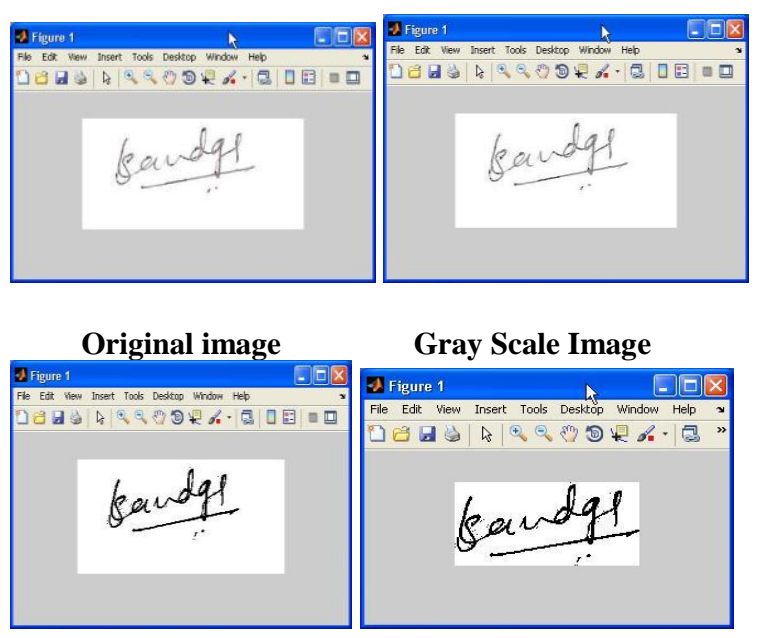

Gray Scale Image

Binary Image Cropped Image

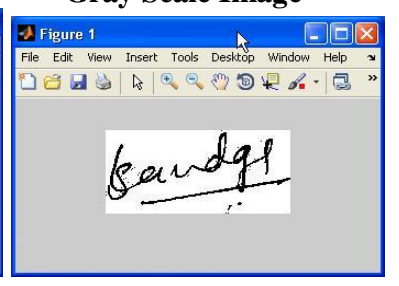

Figure 1: Preprocessing Steps 


\subsection{Feature Extraction}

In offline signature verification, feature extraction is the most important step. Two types of features can be used for signature verification: functions or parameters. For offline signature verification mostly parameter features are used, because in parameter features the signature is characterized as a vector of elements, each element represents the value of a feature. Parameters are generally classified into two main categories: global and local [1]. For parameter feature extraction purpose, we use Krawtchouk Moments [10], [11], [12]. It extract optimal feature from the signature sample depending on the Maximum Entropy Principle, which reduces the input dimensionality of feature vector by eliminating some features with low specified criteria. Krawtchouk moment invariant [10], [11] have desirable properties of being invariant under image scaling, translation and rotation.

Krawtchouk moments are set of moments formed by using krawtchouk polynomials [11] [13] and are a set of polynomials associated with the binomial distribution. The set of moments are rotation, scale and translation invariant. We shall designate these set of moments as Krawtchouk moment invariants. some examples of Krawtchouk moment invariants are as follows:

$$
\begin{gathered}
\tilde{Q}_{n m}=[\rho(n) \rho(m)]^{-\frac{1}{2}} \sum_{i=0}^{n} \sum_{j=0}^{m} a_{i, n, p_{1}} a_{j, m, p_{2}} \tilde{V}_{i j} \\
\tilde{Q}_{00}=\Omega_{00} \tilde{V}_{00} \\
\tilde{Q}_{10}=\Omega_{10}\left[\tilde{V}_{00}-\frac{1}{(N-1) P_{1}} \tilde{V}_{10}\right] \\
\tilde{Q}_{01}=\Omega_{01}\left[\tilde{V}_{00}-\frac{1}{(N-1) P_{2}} \tilde{V}_{01}\right] \\
\tilde{Q}_{11}=\Omega_{11}\left[\tilde{V}_{00}-\frac{1}{(N-1) P_{1}} \tilde{V}_{10}\right] \\
-\Omega_{11}\left[\tilde{V}_{00}-\frac{1}{(N-1) P_{1}} \tilde{V}_{01}+\right. \\
\left.\frac{1}{(N-1)^{2} p_{1} p_{2}} \tilde{V}_{11}\right]
\end{gathered}
$$

Where

$$
\Omega_{n m=\left[\rho\left(n ; p_{1}, N-1\right) \rho\left(m ; p_{2}, N-1\right)\right]^{-1 / 2}}
$$

$\tilde{Q}_{n m}$ are the krawtchouk moment invariants.

\subsection{Feature Optimization}

The genetic algorithm is a model of machine learning which derives its behavior from a metaphor of some of the mechanisms of evolution in nature. Since Genetic Algorithms were designed to efficiently search large spaces and they have been used for number of application such as camera calibration [14], signature verification [15], medical diagnosis [16], facial modeling [17] and handwritten recognition [18]. In many applications, it is seen that system generates hundreds of features. However, it has been observed that, beyond a certain point, the inclusion of additional features leads to a worse rather than better performance. It can also affects the several aspects of the pattern recognition problem such as accuracy, required learning time and necessary number of samples [19]. The main goal of feature subset selection is to reduce the number of features used in classification, while maintaining acceptable classification accuracy. To optimize the features, which are generated by the feature extraction method can enhance the performance of the system. In the optimization phase, Simple Genetic Algorithm (SGA) is used. The following figure shows how the SGA cycle is working.

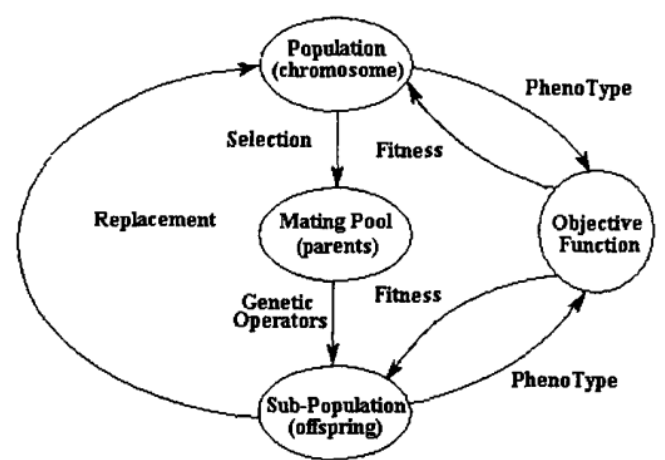

Figure 2: A SGA Cycle

In this experiment SGA is used, i.e., an algorithm based on bit representation, one-point crossover, bit-lip mutation, roulette wheel selection (with elitism). First we initialize the population. We have inserted a chromosome with all features selected. Since we know an admissible solution of the system, it is very interesting to use such knowledge in order to speed up the convergence time of the genetic algorithm [20].

The basic operation of genetic algorithm is outline as follows:

Procedure

Begin

$\mathrm{t}<-0$

Initialize $\mathrm{p}(\mathrm{t})$

While (not termination condition)

$\mathrm{t}<-\mathrm{t}+1$

Select $p(t)$ from $p(t-1)$

Crossover $\mathrm{p}(\mathrm{t})$

Mutate $\mathrm{p}(\mathrm{t})$

Evaluate $\mathrm{p}(\mathrm{t})$

End

End

\subsection{Classification}

The classification phase is required for training and testing purpose. Architecture shown in figure-3 is consist of four layers and it is divided into the three subsections named as Main section, Overlap Compensation Neuron section (OCN), 
and Containment Compensation Neuron section $(\mathrm{CCN})$ as it called Compensatory Neuron Compensation block [21].

In OCN, the neuron represent in this subsection is a hyperbox of size equal to the overlap region between two hyperboxes belonging to different classes. OCN Produces two outputs, one each for the two overlapping classes. OCN is active only, when a test sample belongs to the overlap region.

The CCN is trained to handle the overlap in the pattern area. $\mathrm{CCN}$ represents an overlapping region in the hyperbox from different classes. This neuron is active only when test data sample falls inside the containment region. The output of this neuron is connected to the class that contains the hyperbox of other class. The hyperbox node in $\mathrm{CCN}$ section is created whenever hyperbox of one class is contained within a hyperbox of other class. The connection between the hyperbox and class node are represented by $\mathrm{Z}$ matrix as represented in the figure 3 .

\subsubsection{Training Algorithm}

The training algorithm has mainly two step process hyperbox creation and expansion/creation of compensatory neuron, if overlap exits which is explained in [21]. Let the training starts with the input sample is $A_{h}$. The hyperbox is created for a ordered pair and if the pattern is not fall in the hyperbox then existing hyperbox is expanded with the criteria,

$$
n \theta \geq \sum_{i=1}^{n}\left(\max \left(w_{j i}, a_{h i}\right)-\min \left(v_{j i}, a_{h i}\right)\right)
$$

Where $\theta$ is an expansion coefficient

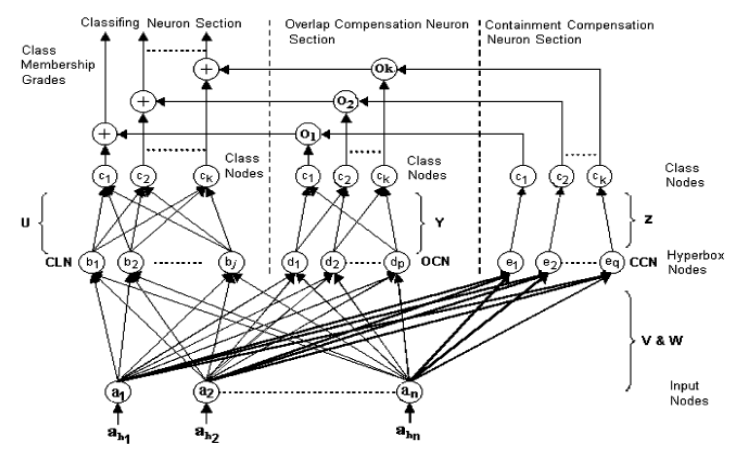

Figure 3: Architecture of FMCN

Also, if the hyperbox is not overlapping with any previous hyperbox of different classes. Then the min and max point of hyperbox is adjusted as

$V_{j i}^{\text {new }}=\min \left(V_{j i}^{\text {old }}, a_{\text {hi }}\right)$ for $i=1,2 \ldots \ldots n$

$$
W_{j i}^{\text {new }}=\max \left(W_{j i}^{\text {old }}, a_{h i}\right) \text { for } i=1,2, \ldots \ldots n
$$

The second step is a creation of compensatory neuron. The isolation test is carried out which checks

$$
\left(w_{j i}<v_{k i}\right) \operatorname{or}\left(w_{k i}<v_{j i}\right)
$$

for any value of $i$, indicates that two hyperbox $\left(b_{j}, b_{k}\right)$ are isolated. So, no compensation is needed and takes new signature sample for training. Otherwise, the containment test will be carried out. In this containment test if

$$
\left(v_{j i}<v_{k i}<w_{k i}<w_{j i}\right) \operatorname{or}\left(v_{k i}<v_{j i}<w_{j i}<w_{k i}\right)
$$

then we can say that the hyperbox $b_{j}$ contained in hyperbox $b_{k}$ or hyperbox $b_{k}$ contained in hyperbox $b_{j}$ respectively.

Then a new CCN node is created in the neural network and hyperbox min and max points are

$$
\begin{aligned}
& V_{c i}=\max \left(v_{k i}, v_{j i}\right) \text { for } i=1,2, \ldots \ldots n \\
& W_{c i}=\min \left(w_{k i}, w_{j i}\right) \text { for } i=1,2, \ldots \ldots n
\end{aligned}
$$

Then take a new signature sample for training and if this condition is not satisfied, the overlap compensation neuron is created because the hyperbox is not isolated or contained. The dimension for hyperbox representing the compensatory neuron are given by,

$$
\begin{aligned}
& V_{o i}=\max \left(v_{k i}, v_{j i}\right) \text { for } i=1,2, \ldots \ldots n \\
& W_{o i}=\min \left(w_{k i}, w_{j i}\right) \text { for } i=1,2, \ldots \ldots n
\end{aligned}
$$

\section{LITERATURE SURVEY}

In this section we have studied the various research papers for signature verification system, feature extraction, and feature optimization techniques. We have studied the research work carried out by various researchers and some of the important research works are as follows:

Bortolozi et. al. [22] uses the graphometric features which are static features like the density of pixels and the pseudo dynamic features represented by axial slant. They employ grid segmentation and divide the signature image into four zones each with column containing cells with horizontal and vertical projections. Each column is converted to a characteristic vector assigned a numeric value. A HMM is used for the learning and verification process.

Faez et. al. [23] uses global features of the signature like the skeleton of the pen trace and the structure of upper and lower envelope are used as shape descriptors. These are obtained by sampling upper and external points from the binary image of the signature. High pressure regions where the writer made more pressure or emphasis to be generated to a linear function that is be used for maximizing the correlation between the vertical and horizontal projections of the skeleton. For each of the above shape descriptors a multi- layer perception is assigned and the network is trained with a modified back propagation algorithm and the output of each individual network is combined through a fuzzy integral voter. Using a test set of 1000 signatures the approach obtained $90 \%$ true verification.

Blumenstein et. al. has proposed system [24] which uses structure features from the signatures contour, modified direction feature and additional features like surface area, length skew and centroid feature in which a signature is divided into two halves and for each half a position of the center of gravity is calculated in reference to the horizontal axis. For classification and verification two approaches are compared the Resilient Back Propagation (RBP) neural network and Radial Basic Function (RBF) using a database of 2106 signatures containing 936 genuine and 1170 forgeries. These two classifiers register $91.21 \%$ and $88 \%$ true verification respectively.

Abuhaiba [25] has avoids the use of features and uses only raw binary pixel intensities. Offline $\mathrm{HSV}$ problem is formulated as graph matching problem. A binary image is 
represented as graph with a set of vertices and edges, the goal is to get the minimum cost of matching which is represented as a classic form of assignment problem in graph theory. The method tested 75 signatures for skilled forgery and 300 signatures for random forgery. This reports $26.7 \%$ and $5.6 \%$ FAR, 26.7\% and 5.6\% EER for skilled and random forgeries respectively.

The uniqueness of writers' handwriting is mapped with that of the signature by Srihari et.al. [26]. The writer sign in a predefined space of $2 \times 2$ inches and rotation is normalized with the horizontal axis. The gradient, structural and concavity are used as image descriptors. The gradient detects the local features of the image and the concavity detects the relationship between the structural and the local features. The verification model is based on the Bayesian classifier is that uses mean and variance measures to classify. The system use two databases of signature with a total of 106 writers and 3960 samples and obtain FRR of $21.90 \%$ and $30.93 \%$ respectively.

Reddy et. al. [27] has proposed the method of geometric centre for feature extraction. The centre is obtained through vertical and horizontal splitting of the image. The signatures used are taken at different time periods to show the intrapersonal variations. The classification is done through a Euclidean classifier model, which is a measure of variance between any two image vectors. For testing 21 genuine signatures and 30 forgeries are used. A set of 9 signatures is used for training the model, FAR obtained are $2.08 \%, 9.75 \%$ and $16.36 \%$ for random, simple and skilled forgeries respectively. The FRR for original signatures is $14.58 \%$.

Enturk, et.al [28] uses Support Vector Machine (SVM). SVM is machine learning algorithms that use a high dimensional feature space and estimate differences between classes of given data to generalize unseen data. The system uses global, directional and grid features of the signature and SVM for classification and verification. The database of 1320 signatures is used from 70 writers. 40 writers are used for training with each signing 8 signatures thus a total of 320 signatures for training. For initial testing the approach uses 8 original signatures and 8 forgeries achieves FRR 2\% and FAR $11 \%$.

Shashi Kumar et. al. [29] has presented the Off-line Signature Verification Based on Fusion of Grid and Global Features Using Neural Networks (SVFGNN). The global and grid features are fused to generate set of features for the verification of signature. The test signature is compared with data base signatures based on the set of features and match/non match of signatures is decided with the help of Neural Network. The performance analysis is conducted on random, unskilled and skilled signature forgeries along with genuine signatures.

Chen et. al. [30] has uses a sequence of data is obtained by tracing the exterior contour of the signature which allows the application of string-matching algorithms. The upper and lower contours of the signature are first determined by ignoring small gaps between signature components. The contours are combined into a single sequence so as to define a pseudo-writing path. To match two signatures a non-linear normalization method, viz., dynamic time warping, is applied to segment them into curves. Shape descriptors based on Zernike moments are extracted as features from each segment. A harmonic distance is used for measuring signature similarity. Performance is significantly better than that of a word-shape based signature verification method. When the two methods are combined, the overall performance is significantly better than either method alone. With a database of 1320 genuine and 1320 forgeries the combination method has an accuracy of $95 \%$ (with $20 \%$ rejection) which is comparable to that of on-line systems.

From literature survey so far, we come to conclusion that that most of the researches have work on three phases of signature verification system i. e. Preprocessing, Feature Extraction and Classification till now. In feature extraction, sometimes unnecessary or redundant features are also generated. Due to these features, system can lead to a worse performance. So we can optimize these features by adding a new phase as Feature Optimization. By adding this phase features will be optimized and required learning time will be also less for classification phase.

\section{PROPOSED METHODOLOGY}

Figure - 4 gives a flow of proposed system. Initially, input signature is taken on a paper then that signature scanned through the scanner and softcopy of input signature is generated. After getting the softcopy, preprocessing is performed on the input signature. After preprocessing, the feature of the processed input signature image is extracted and stored in the form of finite vectors. If the numbers of finite vectors are more, we optimize them by using the Genetic Algorithm. The training samples of these vectors are generated in training stage and finally by using these samples classification phase classify it into classes and generate the result after matching it with the database. As a result, classification phase shows to which person the signature belongs.

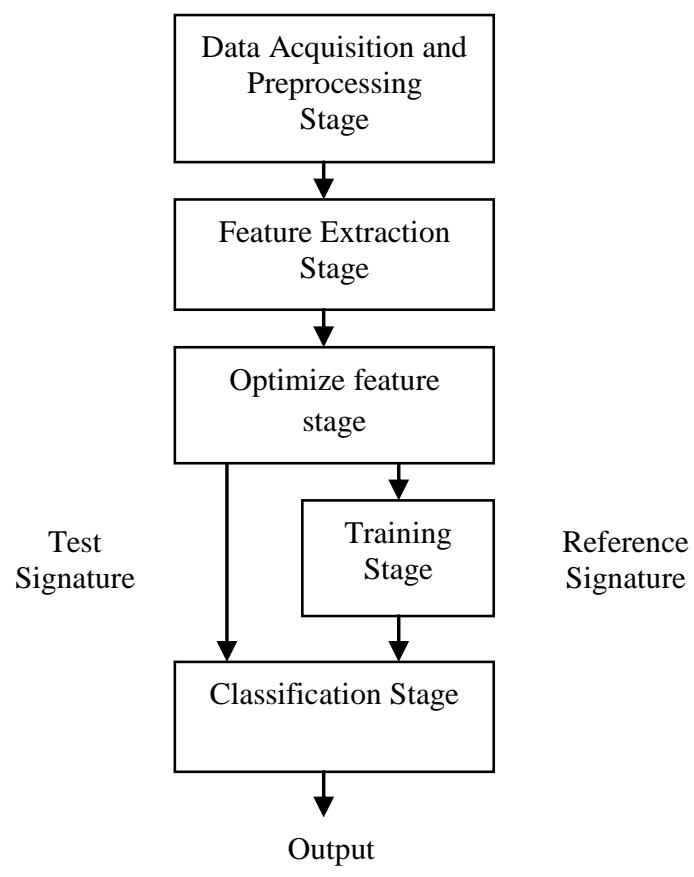

Figure 4: The flow of Offline Signature Verification System

The proposed system has the following steps:

Data Acquisition Stage: In this stage the signature samples or patterns are collected through the scanner. 
Preprocessing Stage: Preprocessing includes various operations like Gray Scaling, Binarization and Outline Calculation.

In gray scaling, original signature image is converted into a Gray Scale Image. A grayscale image is simply one in which the only colors are shades of gray. The reason for differentiating a color image into a gray scale image is that less information needs to be provided for each pixel which will help in generating binary images.

A binary image is a digital image that has only two possible values for each pixel. Typically two colors are used for a binary image such as black and white. When image is a binary image, less information is needed to be provided for a pixel which is helpful in outline calculation and extracting the features of the image.

Feature Extraction Stage: Feature extraction is a special form of dimensionality reduction. Transforming the input data into the set of features is called feature extraction. If the features extracted are carefully chosen, it is expected that the features set will extract the relevant information from the input data. For feature extraction we have used the krawtchouk moment which is invariant to shifting, rotation and scaling. Finite vectors are obtained as a result of feature extraction.

Feature Optimization Stage: In feature optimization stage, we are optimizing the features obtained from krawtchouk moment invariant. Beyond a certain point, the inclusion of additional features leads to a worse rather than better performance. It can also affects the several aspects of the pattern recognition problems such as accuracy, required learning time and necessary number of samples. Feature optimization is used to reduce the number of features used in classification while maintaining acceptable classification accuracy.

Training Stage: In training phase, characteristic properties of typical image features are isolated and, based on these, a unique description of each classification category, i.e. training class, is created. Before the execution of above steps for verification of signature, we have to train the database for generating the training samples. The samples of the input signature are also generated and using FMCN these input signature samples are matched with the trained samples are matched. By using the training sample, FMCN classify the signature into different classes. For training stage we use FMCN training algorithm explained in section II.

Classification Stage: The final stage of proposed system is classification, where the matching of input signature and database signature is done. Classification includes a broad range of decision-theoretic approaches to the identification of images. Image classification analyzes the numerical properties of various image features and organizes data into categories. In testing phase, feature-space partitions are used to classify image features. For classification Fuzzy Min Max Neural Network with Compensatory Neuron is used.

\section{IMPLEMENTATION AND RESULT}

The proposed Signature Verification System is implemented by using MATLAB. For database we have taken signatures of 50 persons. These persons are from various domains like teachers, students, workers, housewives, businessmen even the illiterate persons. We take 10 signatures of each person in various manners. In this way we have collected 500 signatures. The proposed system is evaluated on two performance basis. First is feature extraction and second is time required for classification after optimizing the features. The table--1 shows the 10 signatures samples of a single person.

Table 1: Signature Samples of One Person

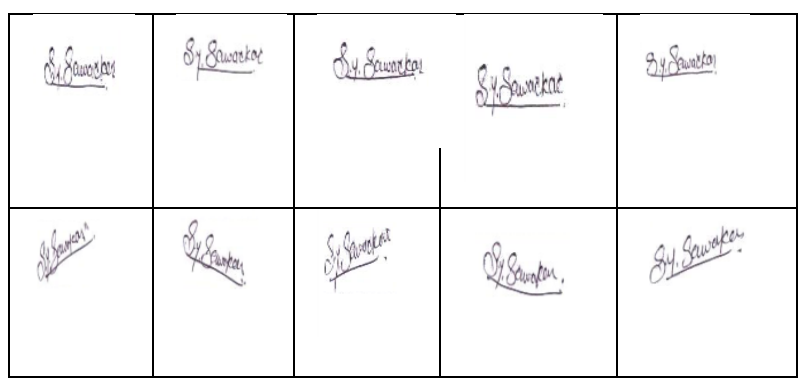

To measure the comparative accuracy of the proposed system, performance factor FRR (false rejection rate) and TAR (true acceptance rate) is used. Therefore Table-2 represents performance of the Offline Signature Verification system in terms of True Acceptance Rate (TAR) and False Rejection Rate (FRR). The True Acceptance Rate is the percentage of original signatures which system accepts. The False Rejection Rate is the percentage of original signatures which system rejects.

Table 2: Comparison of Performance between HU moments and Krawtchouk Moments used in Offline Signature Verification

\begin{tabular}{|c|c|c|c|c|c|}
\hline \multicolumn{2}{|c|}{$\begin{array}{c}\text { Inputs Test } \\
\text { Signatures }\end{array}$} & \multicolumn{2}{c|}{$\begin{array}{c}\text { Accepted/ } \\
\text { Rejected } \\
\text { Signatures }\end{array}$} & \multicolumn{2}{c|}{$\begin{array}{c}\text { Performance } \\
\text { Metrics \% }\end{array}$} \\
\hline $\begin{array}{c}\text { HU Moment } \\
\text { with GA }\end{array}$ & 120 & Accepted & 103 & TAR & 85.83 \\
\cline { 3 - 6 } & Rejected & 17 & FRR & 14.16 \\
\hline $\begin{array}{c}\text { Krawtchouk } \\
\text { Moment } \\
\text { with GA }\end{array}$ & 500 & Accepted & 499 & TAR & 99.8 \\
\cline { 3 - 6 } & Rejected & 1 & FRR & 0.2 \\
\hline
\end{tabular}

The value of TAR for the HU Moments with optimized feature using Genetic Algorithm is $85.83 \%$, while for the Krawtchouk Moment with optimized features using Genetic Algorithm is $99.8 \%$. The value of FRR for the HU Moments with optimized feature using Genetic Algorithm is $14.16 \%$, while for the Krawtchouk Moment with optimized features using Genetic Algorithm is $0.2 \%$.

As we can see in Table-1 a single person has made ten signatures in various manners and various positions. When we extract the features of such signature, the feature vectors can be different for each signature though all signatures belong to single person. To solve this problem, we are using Krawtchuok moment invariant for Feature Extraction Purpose. Krawtchouk moment have two strong characteristics: Invariance and reconstruct-ability. The krawtchouk moments are invariant to Shift, Scale and Rotation. The ability to reconstruct signature from their extracted features ensures that complete information about the 
signature shape is present in these features. The krawtchouk moments are orthogonally stable. Krawtchouk moments also have the interesting property of being able to extract local features of an image.

Therefore the result shows that Krawtchouk moment invariant with optimized feature using Genetic Algorithm gives more efficient feature vectors as compared to HU's moment invariant with optimized feature using Genetic Algorithm.

The graph shown in the figure 6.1 and 6.2 depicts the result in terms of FRR and TAR. The overall performance of the signature recognition system is calculated with respect to the signature pattern database. This database is used to train the Fuzzy Min Max Neural Network with Compensatory Neuron.

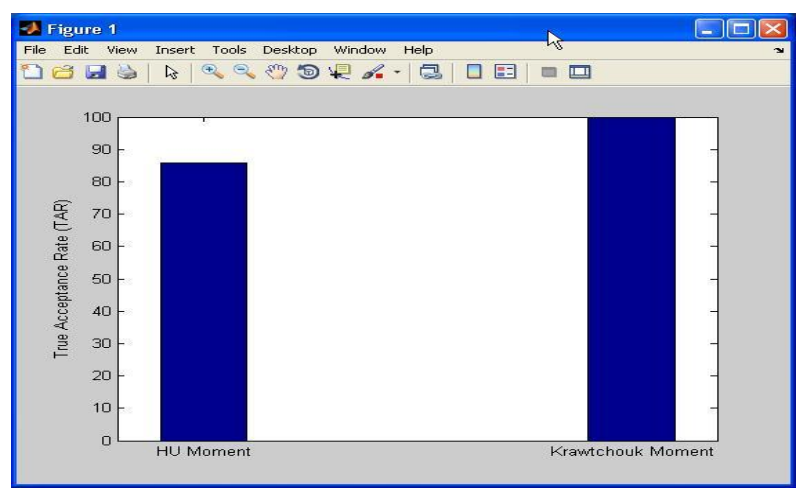

Figure 6.1: True Acceptance Rate (TAR) of previous HU moment with feature optimization using Genetic Algorithm and Krawtchouk moment with feature optimization using Genetic Algorithm

With the above results, after extracting the features of the signature, we are optimizing the features before using it for classification phase. When features are extracted, some redundant features are generated. Due to these redundant features, system takes more time for training as well as for classification. To solve this problem we optimize these features using Genetic Algorithm. There are two advantages of optimizing the features. First, the redundant features are optimized and second the time required for classification is less as compared to not optimizing the features using Genetic Algorithm.

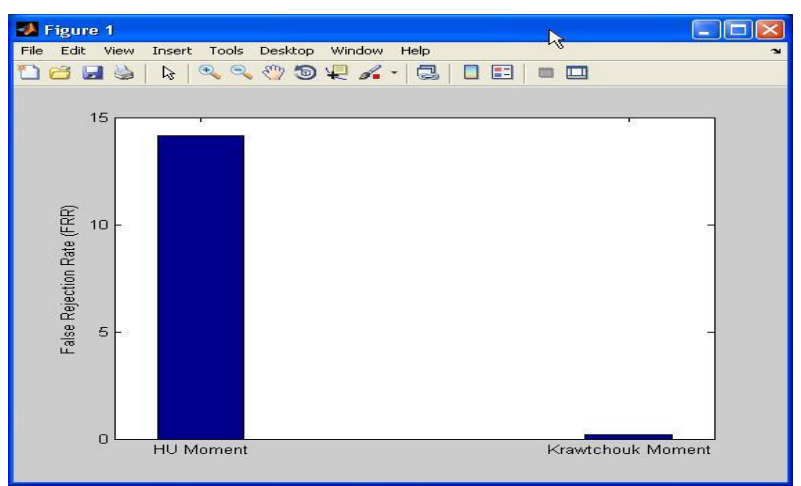

Figure 6.2: False Rejection Rate (FRR) of previous HU moment with feature optimization using Genetic Algorithm and Krawtchouk moment with feature optimization using Genetic Algorithm

\section{CONCLUSION}

In this paper, Krawtchouk moment invariant is used with Genetic Algorithm and Fuzzy Min Max Neural Network with Compensatory Neuron. As Krawtchouk moments are invariant to scaling, rotation and translation it gives highest classification rate as compared to other feature extraction methods. The Krawtchouk Moment with optimized features using Genetic Algorithm gives 99.8\% accuracy. Genetic Algorithm is used for optimization of the extracted features. However, it has been observed that, beyond a certain point, the inclusion of additional features leads to a worse rather than better performance. It can also affects the several aspects of the pattern recognition problem such as accuracy, required learning time and necessary number of samples. Optimizing the features substantially reduces the computational burden from the classifier stage. Therefore the time required for classification stage is less.

\section{REFERENCES}

[1] Donato Impedovo, and Giuseppe Pirlo, "Automatic Signature Verification: The State of the Art", IEEE Transactions on Systems, Man, and Cybernetics-Part C: Applications and Reviews, Vol. 38, No. 5, September 2008.

[2] Q. Yingyong, B. R. Hunt, "Signature Verification using Global and Grid Features", Pattern Recognition, Vol. 27, No.12, pp.1621-1629, Dec.1994.

[3] J. P. Drouhard, R. Sabourin, and M. Godbout, "A Neural Network Approach to Off-line Signature Verification using Directional PDF", Pattern Recognition, Vol. 29, No. 3, pp. 415-424, March 1996.

[4] G. Rigoll, A. Kosmala, "A Systematic Comparison between On-Line and Off-Line Methods for Signature Verification with Hidden Markov Models", 14th International Conference on Pattern Recognition - Vol II, Australia, pp. 1755-1757, 1998.

[5] M. C. Fairhurst, "Signature Verification Revisited: Promoting Practical Exploitation of Biometric Technology", Inst. Elect. Eng. Electron. Commun. Eng. J. (ECEJ), Vol. 9, No. 6, pp. 273-280, Dec. 1997.

[6] M. C. Fairhurst and E. Kaplani, "Perceptual Analysis of Handwritten Signatures for Biometric Authentication", Inst. Elect. Eng. Proc. Vis., Image Signal Process., Vol. 150, No. 6, pp. 389-394, Dec. 2003.

[7] F. Leclerc and R. Plamondon, "Automatic Signature Verification: The State of the Art-1989-1993", Int. J. Pattern Recognit. Artif. Intell. (IJPRAI), Vol. 8, No. 3, pp. 643-660, Jun. 1994.

[8] G. Pirlo, "Algorithms for Signature verification", in Proc. NATO-ASI Series Fundamentals in Handwriting Recognition, S. Impedovo, Ed.Berlin, Germany: Springer-Verlag, , pp. 433-454, 1994.

[9] R. Plamondon and G. Lorette, "Automatic Signature Verification and Writer Identification The state of the art”, Pattern Recognition., Vol. 22, No. 2, pp. 107-131, Jan. 1989. 
[10] M. Krawtchouk, "On Interpolation by Means of Orthogonal Polynomials", Memoirs Agricultural Inst. Kyiv, Vol. 4, pp. 21-28, 1929.

[11] P. T. Yap, P. Raveendran, and S. H. Ong, "Krawtchouk Moments as a New Set of Discrete Orthogonal Moments for Image Reconstruction", IJCNN'02, Vol. 1, pp. 908912, May.2002.

[12] Anass El affar, Khalid Ferdous, Abdeljabbar Cherkaoui, Hakim El fadili, and Hassan Qjidaal, "Krawtchouk Moment Feature Extraction for Neural Arabic Handwritten Words Recognition", International Journal of Computer Science and Network Security (IJCSNS), Vol.9, No.1, pp. 443-448, Jan.2009.

[13] G. Szego, "Orthogonal Polynomials", American Mathematical Society, Colloquium publication, Vol. 23, $4^{\text {th }}$ Edition, 1975

[14] Q. Ji, and Y. Zhang, "Camera Calibration With Genetic Algorithms", IEEE Transactions on Systems, Man, and Cybernetics, Part A: Systems and Humans, Vol. 31, No. 2, pp. 120-130, March 2001.

[15] V. E. Ramesh and N. Murty, "Off-Line Signature Verification Using Genetically Optimized Weighted Features", Pattern Recognition, Vol. 32, No. 2, pp. 217-233, Feb.1999.

[16] J. Yang and V. Honavar, "Feature Subset Selection Using a Genetic Algorithm", IEEE Intelligent Systems, Vol. 13, No. 2, pp. 44-49, Apr.1998.

[17] S. Y. Ho and H. L. Huang., "Facial Modeling From an Uncalibrated Face Image using a Coarse-To-Fine Genetic Algorithm", Pattern Recognition, Vol. 34, No. 5, pp. 1015-1031, May.2001.

[18] G. Kim and S. Kim, "Feature Selection Using Genetic Algorithms for Handwritten Character Recognition", 7th IWFHR, pp. 103-112, Sep.2000.

[19] L. S. Oliveira, N. Benahmed, R. Sabourin, F. Bortolozzi, C. Y. Suen, "Feature Subset Selection Using Genetic Algorithm for Handwritten Digit Recognition", IEEE proceedings of XIV Brazilian Symposium, pp. 362-369, Oct.2001.

[20] L. Davis. Handbook on Genetic Algorithms. Van Nostrand Reinhold, 1991.
[21] Abhijeet V. Nandedkar, Prabir K. Biswas, “A Fuzzy Min-Max Neural Network Classifier with Compensatory Neuron Architecture", IEEE transactions on neural networks, Vol. 18, No. 1, pp. 42 -54, Jan.2007.

[22] F. Bortolozi. E. R. Justino., A. E. Yocoubi, and R Sabourin, "An Off-line Signature Verification System Using HMM and Graphometric Features", $4^{\text {th }}$ IAPR International Workshop on Document Analysis Systems, pp. 211-222, 2000.

[23] K. Faez, M. Dehghan, M. Fathi, "Signature Verification Using Shape Descriptor and Multiple Neural Network", Proceedings of IEEE TENCON on Speech and Image Technologies For Computing and Telecommunications, Vol. 1, pp. 415-418, Dec.1997.

[24] M. Blumenstein, S. Armand, Muthukkumarasamy, "Offline Signature Verification using the Enhanced Modified Direction Feature and Neural based Classification", International Joint Conference on Neural Networks, pp. 684-691, Oct.2006.

[25] S. I. Abuhaiba, "Offline Signature Verification Using Graph Matching", Turk J Elec Engine, Vol. 15, No. 1, pp. 89-104, 2007

[26] S. Reddy, B. Maghi, P. Babu Novel, "Features for Offline Signature Verification", International Journal of Computer, Communication and Control, Vol. 1, pp. 1724, Jun.2006

[27] T. S. Enturk. E. O. Zgunduz. and E. Karshgil, "Offline Signature Verification and Recognition by Support Vector Machine", Proceedings of the 13th European Signal Processing Conference (EUSIPCO), Antalya Turkey, Vol. 4, pp. 2370-2373, Sep.2005.

[28] D. R. Shashi Kumar, K. B. Raja, R. K Chhotaray, Sabyasachi Pattanaik, "Off-line Signature Verification Based on Fusion of Grid and Global Features Using Neural Networks", International Journal of Engineering Science and Technology Vol. 2, No. 12, pp. 7035-7044 2010.

[29] Siyuan Chen, and Sargur Srihari, "Use of Exterior Contours and Shape Features in Off-line Signature Verification", Proceedings of the Eighth International Conference on Document Analysis and Recognition ICDAR, Vol. 2, pp.1280-1284, Sep.2005. 